\title{
Inclusión laboral y discapacidad invisible. Hacia una reflexión sobre las barreras y posibilidades del mercado laboral ordinario. El caso de las personas con fibrosis quística en España
}

\author{
Employment inclusion and invisible disability. A reflection on the barriers \\ and possibilities of the ordinary labour market. The case of people with \\ cystic fibrosis in Spain
}

\begin{abstract}
Resumen
Este trabajo tiene como objetivo analizar la situación laboral de las personas con fibrosis quística en España, con qué barreras se encuentran en el acceso y mantenimiento del empleo y qué adaptaciones favorecerían la inserción. Los datos se obtuvieron de una muestra de 368 personas con fibrosis quística residentes en España de entre 16 y 60 años. Se utilizó una metodología mixta combinando encuestas, entrevistas y un grupo de discusión. Los hallazgos sugieren que la rigidez del mercado de trabajo ordinario no protegido y la invisibilidad de la discapacidad, derivada de esta enfermedad, suponen barreras para compatibilizar trabajo y cuidados. Las conclusiones apuntan, como elementos facilitadores para una inserción laboral plena, a factores relacionados tanto con las propias personas con discapacidad como con la estructura del mercado laboral actual.
\end{abstract}

\section{Palabras clave}

Discapacidad, barreras de inserción laboral, teletrabajo, flexibilidad, orientación educativa, fibrosis quística.

\begin{abstract}
The objective of this paper is to review the working conditions of cystic fibrosis patients in Spain and to ascertain obstacles frequently encountered by them in the workplace so as to implement appropriate measures that may facilitate access to work, job security, and comfort for this population. The data was collected from a sample population of 368 people suffering from cystic fibrosis, residing in Spain, and ranging in ages of between 16 and 60 years. The method utilized was a combination of standard questionnaire distribution, personal interviewing and group discussion. Findings suggest an employer inflexibility within the general job market that doesn't make allowances for cystic fibrosis patients and thereby withholds job security from this population as they struggle to balance work with healthcare needs. Conclusions point to necessary adjustments for the individual approach of persons suffering from cystic fibrosis to the job market as well as a restructuring of the job market itself to facilitate access.
\end{abstract}

\section{Keywords}

Disability, barriers to the job market, remote working, flexibility, educational orientation, cystic fibrosis.

\author{
Laura Esteban Romani \\ <laura.esteban@uv.es> \\ Federación Española de Fibrosis \\ Quística. España
}

\section{Mercedes Botija Yagüe <mercedes.botija@uv.es>}

Universidad de Valencia. España

Estefanía Alabau de Lera $<$ comunicacion@fibrosisquistica. org $>$

Federación Española de Fibrosis Quística. España

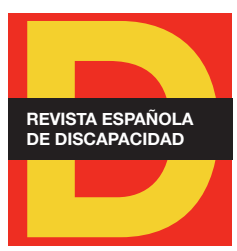

Para citar:

Esteban, L. et al. (2021). Inclusión laboral y discapacidad invisible. Hacia una reflexión sobre las barreras y posibilidades del mercado laboral ordinario. El caso de las personas con fibrosis quística en España. Revista Española de Discapacidad, 9(2), pp. 23-43.

Doi: <https://doi.org/10.5569/23405104.09.02.02>

Fecha de recepción: 31-10-2020 Fecha de aceptación: 04-10-2021 


\section{Introducción}

La fibrosis quística es una enfermedad genética, degenerativa y crónica que afecta principalmente a los pulmones y al aparato digestivo, por lo que no se percibe en el aspecto externo de la persona, pudiendo dar lugar a una "discapacidad invisible" (Syma, 2019, p. 114). Actualmente, se calcula que la fibrosis quística en España tiene una incidencia de 1 cada 5000 nacimientos. Con esta incidencia pasa a catalogarse como enfermedad rara o de baja prevalencia, ya que se consideran como tales aquellas que tienen una prevalencia menor a 1:2000 individuos (Cortés, 2015).

Hace dos décadas, la fibrosis quística era una enfermedad pediátrica, los avances en la medicación y tratamientos han permitido que las personas con fibrosis quística aumenten su esperanza y calidad de vida. El registro nacional elaborado en 2016 contabilizaba un $47 \%$ de personas con fibrosis quística mayores de 18 años y una supervivencia media de 46 años, que aumenta cada año (Vázquez, 2018). Esto hace que la inclusión de las personas con fibrosis quística en el mercado laboral sea una realidad y motivo de estudio. El ámbito laboral se erige en la sociedad contemporánea como un elemento decisivo en el proceso de integración social y participación comunitaria de la ciudadanía, dado su potencial socializador y los ingresos que de él se desprenden (Mercado et al., 2013).

En fibrosis quística, la empleabilidad se ha asociado, además, con puntajes de mejor calidad de vida y menores índices de depresión (Hogg et al., 2006). Diferentes autores (Havermans et al., 2009; LabordeCastérot, 2012) señalan que la asociación positiva entre empleo y algunos parámetros de calidad de vida se mantuvo incluso después de controlar parámetros médicos relevantes.

Ahora bien, no se puede obviar que, en la inserción laboral, las personas con fibrosis quística parten con una serie de desventajas respecto al resto de población, debido a la sintomatología derivada de la enfermedad (Zupanic y Skerjanc, 2019). Las personas con fibrosis quística requieren de un exhaustivo tratamiento diario: una ingesta media de 30 comprimidos, 2 horas de fisioterapia y 1 hora de ejercicio físico (Federación Española de Fibrosis Quística, 2021) y todo ello acompañado de la invisibilidad de sus características (Syma, 2019).

En una investigación sobre la situación psicosocial de las personas con fibrosis quística en España (Federación Española de Fibrosis Quística, 2011), se destacaba que solo el $22 \%$ de las personas con fibrosis quística mayores de 16 años estaba en situación laboral activa, pero no se ofrecían datos concretos sobre el tipo de empleo o la adecuación de este con el mantenimiento de la salud y el tratamiento.

Atendiendo a las particularidades de cada persona, unos empleos son más recomendables que otros para las personas con fibrosis quística (Esteban-Romaní, 2013). Zupanic y Skerjanc (2019) listan los sectores y profesiones no recomendables y en ese listado aparece el sector sanitario debido a la mayor exposición de patógenos respiratorios. Sin embargo, el $10 \%$ de personas con fibrosis quística del estudio de LabordeCastérot (2012) trabajaba en este sector.

Una de las conclusiones del estudio de la Federación Española de Fibrosis Quística (2011) citado en el párrafo anterior destacaba la necesidad de la orientación profesional en la elección de los estudios en las etapas de adolescencia y juventud. Este dato coincide con las conclusiones de varios autores más allá de nuestras fronteras: en Francia, Laborde-Castérot (2012) afirman que muy pocos adolescentes con fibrosis 
quística reciben orientación profesional formal para sus estudios y su posterior inserción laboral. En el estudio de Demars (2011) en Estados Unidos había recibido orientación formal el 1\% de la muestra, mientras que Zupanic y Skerjanc (2019), en una revisión de la literatura sobre orientación educativa en fibrosis quística, constatan que la orientación profesional que reciben los adolescentes y adultos con fibrosis quística en Eslovenia es insignificante.

Los aspectos físicos de la enfermedad crónica pueden afectar a la inserción laboral y a su capacidad para trabajar, pero también existen factores psicosociales, estrategias de afrontamiento y ambiente comunitario que pueden influir. En un estudio con el modelo multivariado se demostró que la situación laboral de las personas con fibrosis quística es más independiente de los parámetros clínicos de la enfermedad que del logro educativo y la percepción subjetiva de la salud (Targett et al., 2014).

El Informe Olivenza 2018 sobre la situación general de la discapacidad en España señala que la invisibilidad de los síntomas de las discapacidades orgánicas, como la fibrosis quística, generan desconocimiento e incomprensión en el entorno, lo cual repercute negativamente en su inserción social (Jiménez, 2018). Además del desconocimiento por parte de la comunidad, se suma lo que señalan otros estudios sobre que las tasas de divulgación de las propias personas con fibrosis quística (sobre su enfermedad en el entorno laboral) son inferiores a las que se producen en el entorno de familiares y amigos, con porcentajes que se acercan al $94 \%$ en entornos familiares, frente al $40 \%$ de divulgación en entornos laborales (Modi et al., 2010).

La mayoría de las investigaciones centran el objeto de estudio en las particularidades de las personas con fibrosis quística y la gravedad de su enfermedad. Edwards y Boxall (2010), sin embargo, aportan la perspectiva del modelo social, que aboga por que no sólo se centren en las experiencias individuales de la enfermedad, ya que estas distraen la atención de las estructuras sociales que acogen estas experiencias.

Larborde-Castérot et al. (2012) certifican que más de la mitad de los pacientes afirmaban estar limitados en el trabajo por su enfermedad y requerían adaptaciones y flexibilidad especialmente en horarios y permisos.

Para compensar dificultades en la inserción, la legislación española regula, en su artículo 40 de la Ley General de derechos de las personas con discapacidad, la existencia del derecho a las adaptaciones en el puesto de trabajo. Ahora bien, es importante apuntar desde el principio que, en el caso de la fibrosis quística y en otros muchos casos de enfermedades con discapacidades orgánicas invisibles, no siempre se obtiene el Certificado de Calificación de Discapacidad (CCD) que otorga el estatus legal de persona con discapacidad. Un estudio de la Federación Española de Fibrosis Quística sobre este certificado y la fibrosis quística (Esteban-Romaní, 2020, p. 79) alerta de que al $15 \%$ de las personas con fibrosis quística que lo ha solicitado no se lo han concedido y, de las que sí lo han recibido, en el $40 \%$ de los casos han tenido que solicitarlo más de una vez. Esta situación hace que la inserción se complique un poco más a la hora de plantear adaptaciones. Aunque es cierto que ya se va teniendo en cuenta esta situación, tal y como señala recientemente Ricardo Esteban-Legarreta (2021) ante el análisis de la sentencia del Tribunal Constitucional 51/2021: "el planteamiento del Tribunal constitucional constituye un espaldarazo a otras resoluciones judiciales que se abren paso tímidamente y que están reconociendo situaciones de discriminación laboral 'por razón' de discapacidad, aunque no concurra en el supuesto una discapacidad evaluada” (p. 321).

En España, la literatura académica concreta, en cuanto a la inserción laboral de las personas con fibrosis quística, es escasa. Este trabajo contribuye a la literatura existente, describiendo cuál es la situación de inserción laboral de las personas con fibrosis quística en España, explorando y analizando cuáles son los 
elementos que dificultan esta inserción y cuáles la podrían facilitar. También trata de ir un paso más allá y visibilizar las opciones de empleabilidad del colectivo de fibrosis quística y la propuesta de adaptaciones en el mercado laboral ordinario, que harían de fibrosis quística-empleo un binomio adaptado. En definitiva, pretende ser una investigación con aplicabilidad práctica. Mary Richmond (citada en Navarro, 2016) ya insistió en que, desde el trabajo social, además de llevar casos individuales, se debía investigar, denunciar los problemas sociales y crear una opinión favorable a la reforma y los avances sociales.

\section{Metodología}

Para alcanzar los objetivos propuestos, se optó por una metodología que combinase técnicas cualitativas y cuantitativas, con el fin de abarcar una visión holística in situ de las percepciones de las propias personas protagonistas. Como instrumentos de investigación, se manejó el cuestionario, la entrevista y el grupo de discusión.

\subsection{Población y muestra}

En España, según la Federación Española de Fibrosis Quística, esta enfermedad crónica y degenerativa afecta a unas 2.500 personas con una incidencia de la enfermedad en nuestro país de 1 persona cada 5000 nacimientos, y se calcula que 1 de cada 35 habitantes son portadores sanos de la enfermedad.

La muestra intencional, no probabilística, se obtuvo al enviar el cuestionario telemáticamente a las 1200 personas mayores de 16 años inscritas en las asociaciones pertenecientes a la Federación Española de Fibrosis Quística. De este envío, se obtuvieron un total de 368 respuestas. Se recopilaron todos los cuestionarios, con independencia del grado de discapacidad, tomando en consideración un concepto amplio de persona con discapacidad, atendiendo a diferentes autores (Botija, 2016; Gutiérrez, 2019; Esteban-Legarreta, 2021).

Para la selección de sujetos en la entrevista semiestructurada y el grupo de discusión se utilizó la técnica bola de nieve con los siguientes criterios de selección: personas con fibrosis quística mayores de 16 años en situación laboral activa o que lo hubiesen estado en algún momento.

Las entrevistas se realizaron a los participantes de la tabla 1 y el número de estos quedó determinado en el momento de saturación de datos, donde las aportaciones ya no revelaban parámetros novedosos esgrimidos por otros participantes. 


\begin{tabular}{l}
\hline Tabla 1. Participantes en la entrevista \\
\begin{tabular}{|l|c|c|c|c|c|}
\hline Cód. & Género & Edad & $\begin{array}{c}\text { Percepción afectación de } \\
\text { la enfermedad }\end{array}$ & $\begin{array}{c}\text { Certificado de Calificación } \\
\text { de Discapacidad (CCD) }\end{array}$ & Palabras \\
\hline Fibrosis quística1 & Hombre & 41 años & Leve & Sin CCD & 2874 \\
\hline Fibrosis quística2 & Mujer & 28 años & Media & $69 \%$ & 5398 \\
\hline Fibrosis quística3 & Hombre & 22 años & Alta & $33 \%$ & 3754 \\
\hline Fibrosis quística4 & Mujer & 37 años & Alta & $67 \%$ & 2734 \\
\hline Fibrosis quística5 & Hombre & 28 años & Media & $69 \%$ & 5835 \\
\hline
\end{tabular}
\end{tabular}

Fuente: elaboración propia.

En el grupo de discusión, se confeccionó un guion dotado de flexibilidad para facilitar el debate discursivo, con la finalidad de corroborar la información obtenida en el cuestionario y las entrevistas, además de generar un discurso de propuestas que facilitara la inserción laboral de las personas con fibrosis quística. La sesión fue conducida por dos personas y grabada en doble formato de audio y vídeo. Contó con 6 participantes, según características de la tabla 2.

Tabla 2. Participantes grupo de discusión
\begin{tabular}{|l|c|c|l|}
\hline Cód. & Edad & Sexo & Perfil laboral \\
\hline GD1-Fibrosis quística & 45 & Hombre & Pensionista por incapacidad laboral \\
\hline GD2-Fibrosis quística & 34 & Mujer & Mantenimiento funcional en potabilizadora \\
\hline GD3-Fibrosis quística & 41 & Hombre & Analista de aplicaciones web \\
\hline GD4-Fibrosis quística & 27 & Mujer & Consultora llunion. Empleo protegido CEE \\
\hline GD5-Fibrosis quística & 28 & Mujer & Farmacéutica, gestión de calidad de producto \\
\hline GD6-Fibrosis quística & 30 & Mujer & Ilustradora. Empleos cortos interrumpidos \\
\hline
\end{tabular}

Fuente: elaboración propia.

\subsection{Instrumentos}

La recogida de información se realizó mediante un cuestionario, entrevistas y grupo de discusión.

Para el análisis cuantitativo se eligió la técnica de cuestionario, atendiendo a algunas de sus principales ventajas, tal y como señala (Díaz de Rada, 2010):

- Posibilidad de adaptar las preguntas a los objetivos de la investigación.

- Facilidad de administración, distintas posibilidades.

- Posibilidad de aplicar la teoría de la probabilidad y el muestreo para poder realizar inferencias en la muestra. 
Se diseñó un cuestionario ad hoc avalado por un grupo de expertos y siguiendo las recomendaciones de Escobar y Cuervo (2008). Este grupo estuvo compuesto por 10 profesionales expertos en integración laboral, discapacidad y fibrosis quística, que contaban con más de 5 años de experiencia laboral en el tema objeto de estudio. Además, el cuestionario se administró como prueba piloto a 5 personas que cumplían las características de la muestra. Con estas aportaciones se construyó el cuestionario definitivo.

El cuestionario estaba compuesto por una primera parte donde se definía el objetivo de este y la identificación y situación educativo-laboral del sujeto que lo rellenaba. Una segunda parte se vinculaba con la enfermedad, grados de discapacidad y trasplante, para, en un tercer apartado, extraer datos sobre elementos obstaculizadores y facilitadores en el ámbito laboral y, por último, obtener información sobre la percepción del sujeto en torno a la inserción laboral. Este instrumento fue administrado entre enero y febrero de 2020.

El diseño de la entrevista (ad hoc) se formuló para poder profundizar, desde la experiencia y perspectiva de la propia persona con fibrosis quística, en el análisis de los elementos que dificultan la inserción laboral y cuáles la podrían facilitar. Esta técnica cualitativa es de las más empleadas en los estudios sobre discapacidad y mercado laboral, ya que permite captar vivencias y discursos que posibilitan una mayor comprensión de los procesos sociales (Sánchez, 2016).

El grupo de discusión se plantea como ámbito experimental óptimo para la circulación de discursos. Discursos que adquieren su máximo valor como representación simbólica del grupo, con respecto al fenómeno estudiado, y representan el ideal de grupo (Callejo, 2002). El grupo de discusión que se realizó en esta investigación duró 1 hora y 49 minutos. El objetivo de esta técnica fue verificar los hallazgos obtenidos en las entrevistas y en las encuestas y generar un discurso de propuestas para un escenario favorecedor de su inserción laboral.

\subsection{Procedimiento y garantías éticas}

Los datos obtenidos de los cuestionarios se exportaron al programa informático SPSS_19, con el que se realizó el análisis estadístico.

Tanto las entrevistas como el grupo de discusión se grabaron, transcribieron literalmente de forma naturalista (respetando la jerga propia) y se analizaron utilizando el programa MAXQDA Versión 11 para Windows, para la codificación, la ordenación y la recuperación de segmentos narrativos.

En el proceso de análisis, los criterios fueron temáticos, agrupándose por segmentos de un mismo tema. La clasificación se realizó buscando tópicos de significado y la posterior asignación de un código. Finalmente, se consensuó la línea metodológica de análisis definitivo y los criterios de saturación de los datos.

La utilización de ambas metodologías y distintas técnicas obedece al interés por complementar los resultados obtenidos, así como enriquecer las conclusiones.

La propuesta metodológica gozó de garantías éticas. Las personas que cumplieron los criterios de inclusión para este estudio recibieron previamente la necesaria información y prestaron consentimiento de participación voluntaria por escrito, de acuerdo con la Declaración de Helsinki (Asamblea Médico Mundial, 2013). Para la administración del cuestionario se informó del objetivo y se recogió el consentimiento. Las entrevistas y el grupo de discusión se realizaron garantizando la confidencialidad de las informaciones y 
el tratamiento reservado con fines de investigación, como es el caso. Se aseguró la anonimización de los participantes estableciendo códigos neutros.

Asimismo, se explicó a los y las participantes la posibilidad de interrumpir o rechazar su contribución en cualquier momento.

\section{Resultados}

La estrategia de análisis ha permitido cumplir la finalidad de analizar las características de la inserción laboral de personas con fibrosis quística y explorar las relaciones entre inserción laboral y factores como: Certificado de Calificación de Discapacidad, género, nivel de estudios, comunicación de la fibrosis quística en el lugar de trabajo y discriminación, y así poner en común necesidades y oportunidades relacionadas con el mercado laboral de las personas con fibrosis quística mayores de 16 años.

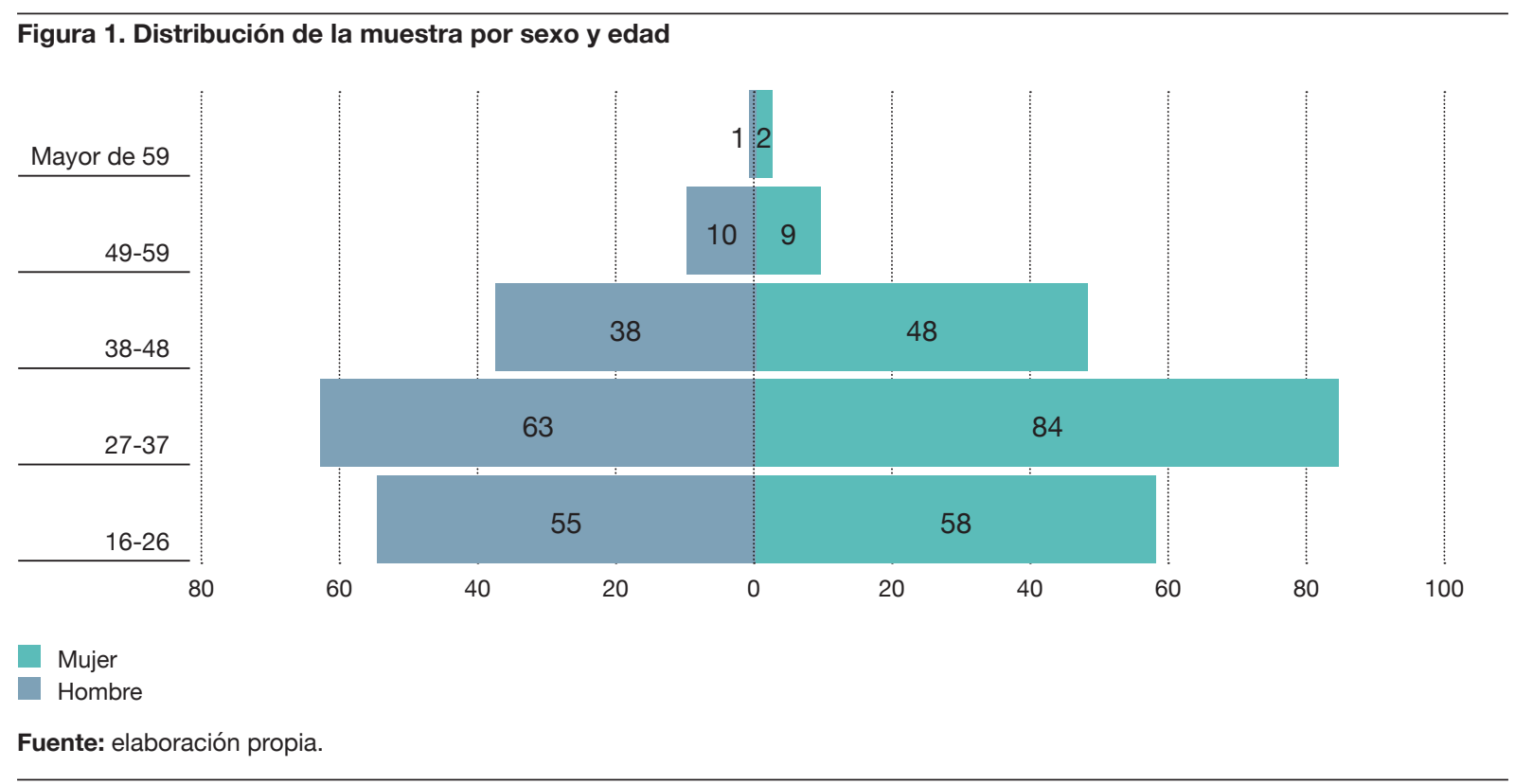

La fibrosis quística es una enfermedad degenerativa que puede desembocar en un trasplante pulmonar. En la muestra objeto de estudio observamos que algo más de la quinta parte está trasplantada $(20,9 \%)$.

El $80 \%$ tiene reconocido el Certificado de Calificación de Discapacidad (CCD) con un grado superior al $33 \%$. 


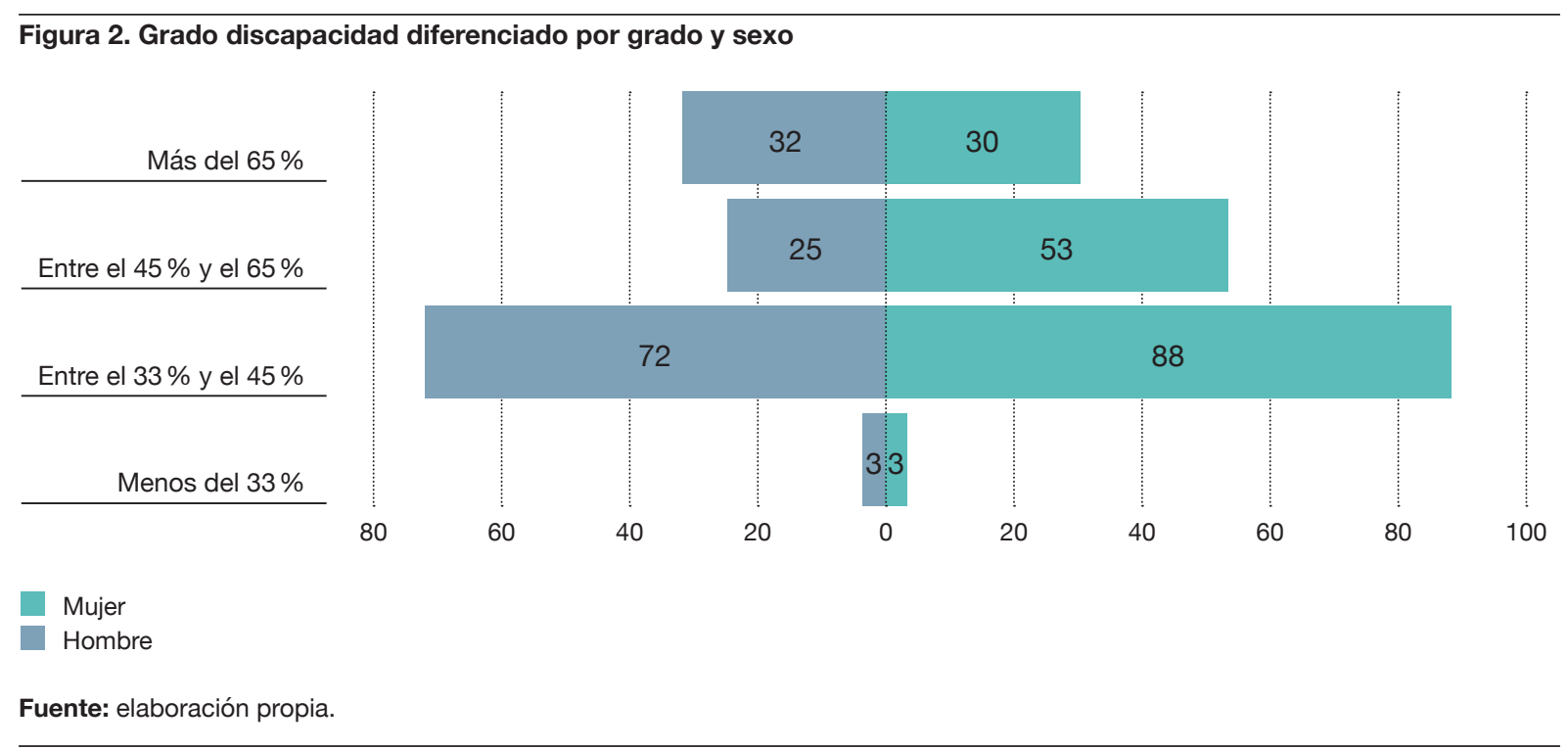

Formación: el nivel de formación de nuestra muestra es del $40 \%$ en referencia a estudios universitarios y las mujeres tienen un nivel de estudios superior al de los hombres.

En cuanto a los tipos de empleo a los que podían optar las personas con fibrosis quística, la elección de los estudios era uno de los aspectos en los que se quería poner el foco de atención, ya que, como aparece citado en la introducción, condicionará el desempeño de la profesión y su mantenimiento. Sorprende que la elección de los estudios, a pesar de lo importante que es para el mantenimiento posterior del empleo, no estuviese presente en su momento. Como se observa en la figura 3, esto no condicionó en la mayoría estadística y se pudo comprobar en el verbatio de los entrevistados cuando respondían a la pregunta "cuándo elegiste los estudios, ¿pensaste en las limitaciones que tiene la fibrosis quística?”.

Pues no lo pensé en ese momento, igual lo debería haber pensado. Cuando tienes que elegir, tienes muchas cosas en la cabeza y en eso no pensé, aunque luego lo descubres igualmente. (Fibrosis quística3).

De primeras, no, la verdad. Tome más la decisión por... no tuve en cuenta ese aspecto... me gustó más por un tema docente y no pensé en si luego esa profesión sería compatible con mi enfermedad. (Fibrosis quística1).

De hecho, son pocas las excepciones donde los propios progenitores o médicos asesoran sobre la dificultad de algunos puestos para las personas que tienen fibrosis quística.

Mi sueño era ser paleontólogo, pero ya desde pequeño me dijeron que, por el tema del polvo, no podría. (Fibrosis quística5). 
En esta misma línea, en el grupo de discusión se observa cómo se han tenido que hacer adaptaciones sobre la formación y las limitaciones concretas que se encontraron.

Yo quería hacer una carrera que sólo estaba en Madrid y, como soy de Guadalajara, pensamos que los traslados no me vendrían bien, me elegí una de aquí, turismo, pero sin pensar en que puedes trabajar en un hotel de cara al público y con turnos... Tuve que reconducir la formación a algo más de oficina. (GD4-Fibrosis quística).

Yo sabía que quería hacer bellas artes sí o sí, sin pensar en nada más, pero cuando la empecé, me di cuenta de que no podía hacer algunas asignaturas, no podía hacer grabado, escultura, pintura en óleo, o sea, mogollón de cosas. Y de repente, tenía que cancelar suscripciones porque me habrían perjudicado los pulmones. Pero bueno, me he tirado por otra vertiente más teórica...(GD6-Fibrosis quística).

Se crea un discurso que es admitido por el grupo, que asiente con la cabeza:

Me hubiese gustado que alguien, como estamos haciendo ahora, me contase su experiencia para poder escoger un poco mejor y no dar tantos tumbos. (GD4-Fibrosis quística).

Empleo: de la muestra, un $75 \%$ tiene o ha tenido trabajo en diferentes ámbitos laborales. La empresa privada es donde se ha realizado este trabajo mayoritariamente (44\%), seguido por la administración pública $(12,2 \%)$, y el tercer sector y el trabajo autónomo, como algo minoritario. Por otra parte, los trabajos realizados a través de centros especiales de empleo sólo suponen el 7,6\%. 
En relación con el empleo, existe un grado de correlación moderado entre hombres y mujeres, según la prueba chi-cuadrado $(0,599)$, mientras que apenas existe dependencia con la posesión del certificado de discapacidad (chi-cuadrado 0,052).

De las 85 personas desempleadas de la muestra, 37 de ellas son estudiantes, pero más de una cuarta parte afirman que no encuentran trabajo debido a la fibrosis quística o a daños colaterales de la misma (incapacidad, jubilación anticipada...). Véase figura 4.

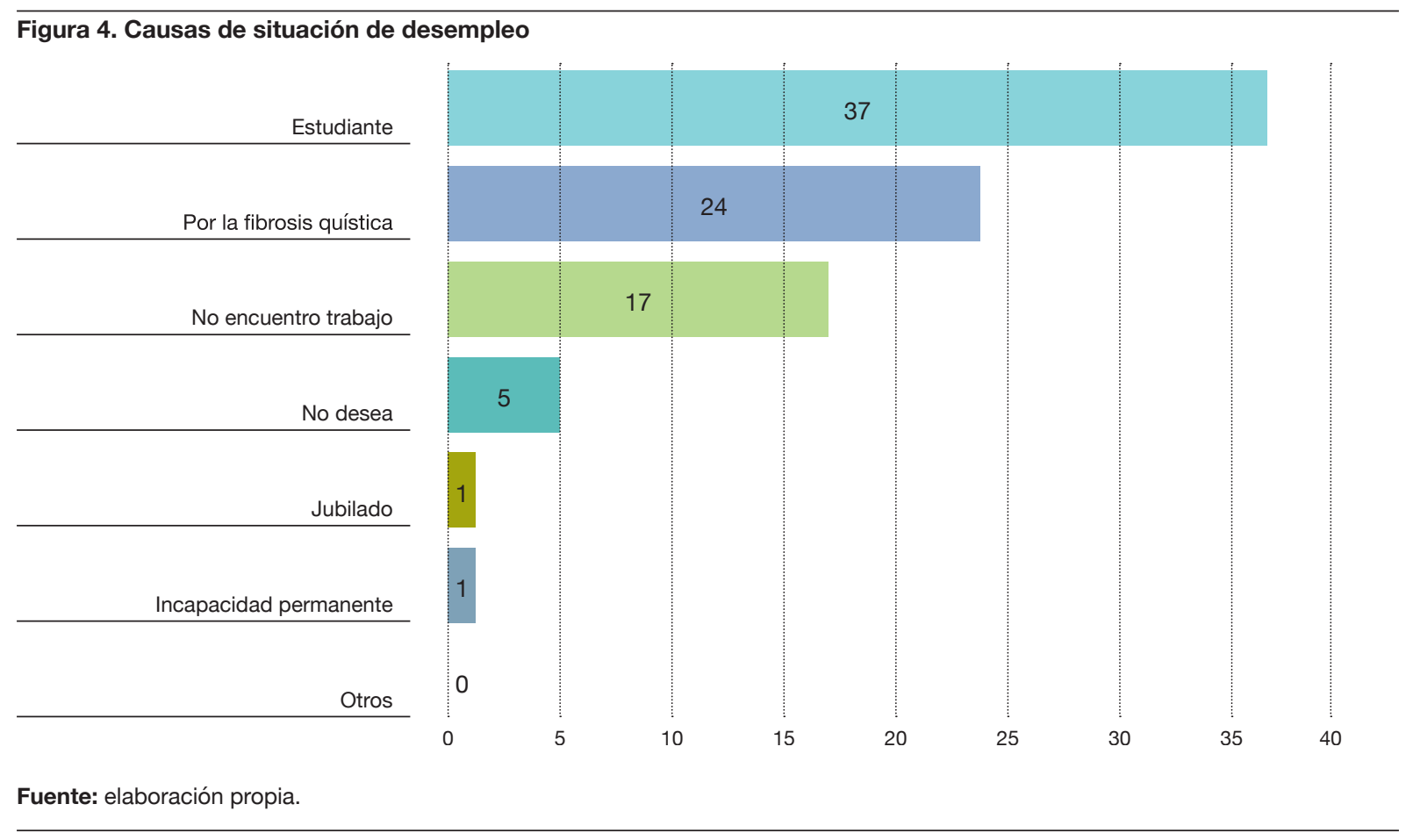

En el grupo de discusión, se diferencia entre los puestos que pertenecen al sector público y de empleo protegido frente a la empresa privada.

Si trabajas para lo público es fabuloso, tus derechos son tus derechos. En la empresa privada parece que tienes que currártelos. (GD3-Fibrosis quística).

He estado en la empresa privada con muchas complicaciones. Ahora mi empresa es un centro especial de empleo, también hay situaciones muy complicadas, pero la diferencia es que no hay incomprensión por parte de los trabajadores ni de los jefes, entonces es un ambiente en el que me siento muy cómoda y además tengo una flexibilidad muy buena. (GD4-Fibrosis quística).

Empecé a trabajar en la universidad haciendo el doctorado, entré por el turno de discapacidad... y pues ... genial, mis directoras son la hostia, conocen la fibrosis quística, me apoyan en todo, me dan flexibilidad... pero yo también curro mucho. (GD6-Fibrosis quística). 
Dificultades vinculadas con el horario: en cuanto a la jornada laboral, la más habitual es la jornada completa (intensiva de mañana o tarde) que supone el 23,9\%, seguida de la jornada completa partida con un 22,3\% y la media jornada de mañana o tarde (9\%). El resto de los porcentajes se distribuye en jornadas sin horario establecido o de fines de semana u horas mensuales, que no se computan dentro de un horario habitual.

Las personas prefieren media jornada, independientemente del grado de discapacidad, aunque expresan la dificultad de que el salario se reduzca a la mitad. Las personas participantes, tanto en las entrevistas como en el grupo de discusión, razonaban la necesidad de la flexibilidad de la jornada.

No pude continuar con mi trabajo, hacía turnos interminables de 24 horas de guardia y eso desbarajusta a cualquier cuerpo, y más a un fibrosis quística. (Fibrosis quística2).

La flexibilidad horaria... sería necesario que las jornadas laborales fueran compatibles con los tratamientos. No sólo porque son largos, sino también porque te dejan muy cansado. Para mí la dificultad más grande que veo es la jornada laboral que existe en la actualidad tan larga. 8 horas son muchas horas para trabajar, pero claro, si trabajas menos, no ganas ná. (Fibrosis quística 3).

Es muy difícil compatibilizar en horarios normales, como viene establecido en las empresas, hace algunos años todavía era peor, pero porque no había tanta flexibilidad. Todo a través del sacrificio personal y económico, o levantarte a las 4:30 de la mañana para compatibilizar tratamiento y horas de trabajo o reducirte la jornada y cobrar la mitad. (GD4-Fibrosis quística).

Condiciones laborales: la estadística nos indica que un 54,7\% de las personas con fibrosis quística tiene faltas mensuales al trabajo. Más del $22 \%$ falta más de 3 veces al mes. Estadísticamente estos porcentajes no tienen relación con el Certificado de Calificación de Discapacidad, como evidencia la falta de asociación entre absentismo y Certificado de Calificación de Discapacidad (figura 5), ya que el coeficiente chi-cuadrado es 0,048.

\section{Figura 5. Absentismo según grado de discapacidad}

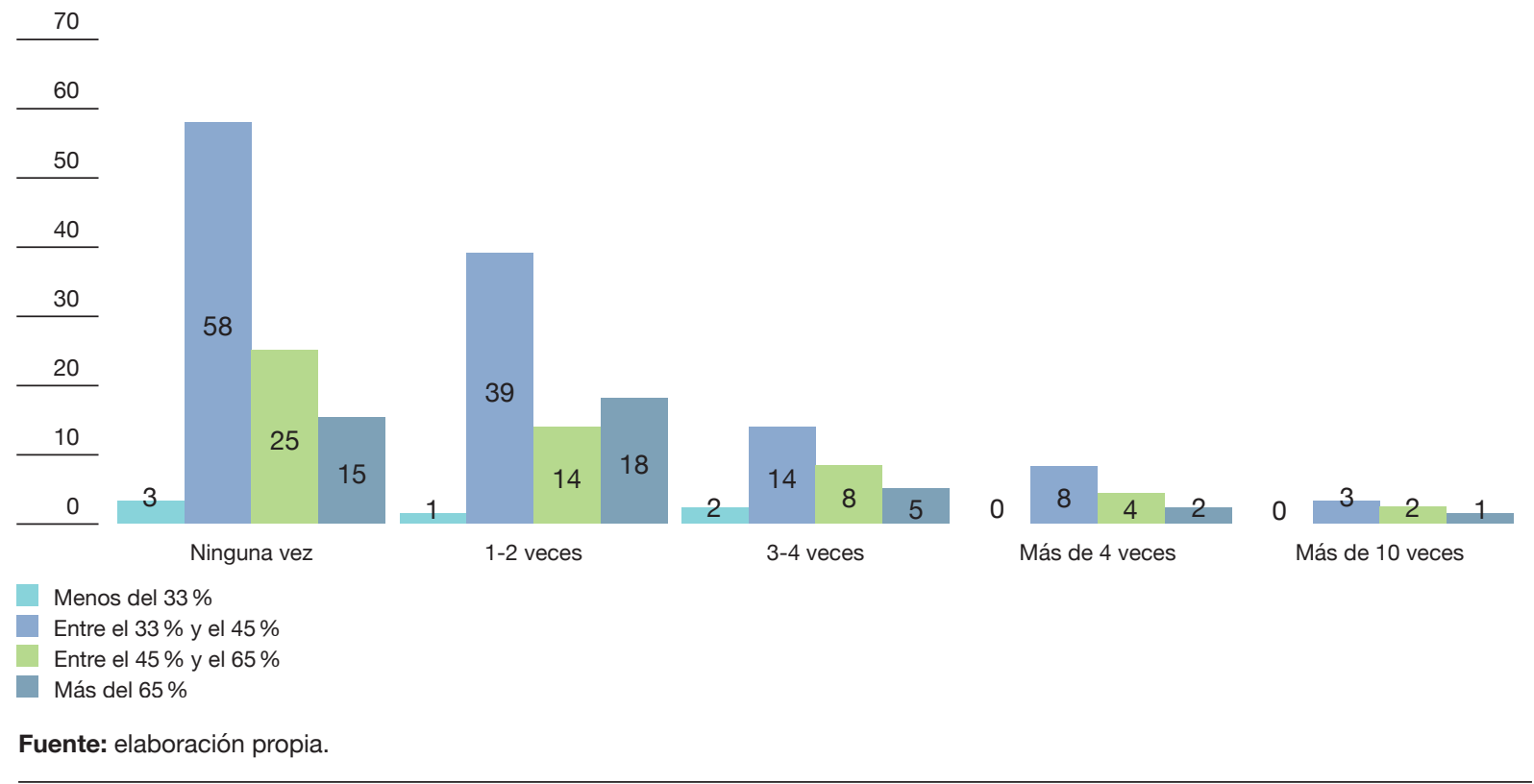


Sin embargo, en los discursos se refleja la dificultad de las personas con relación a las ausencias al trabajo debido a la fibrosis quística:

Al poco de estar trabajando tuve la mala suerte de que tuve que ponerme un intravenoso. Acabó mi contrato y no hubo renovación ¿casualidad? (Fibrosis quística2).

Cuando tenía que faltar por controles médicos, me esforzaba mucho cambiando turnos, horarios, para que no se notaran mis ausencias, pero no servía, yo notaba que no lo entendían, ni mis jefes, ni mis compañeros. (Fibrosis quística4).

Cuando empiezas a trabajar, si tienes que pillar más días de baja porque tienes médicos, analíticas o lo que sea, es ahí donde no entienden el problema. (Fibrosis quística5).

Los factores exógenos en los que se desenvuelven las personas en su ámbito profesional suponen igualmente un obstáculo sustancial:

La principal dificultad que encuentro son las profesiones que provocan desorden de horarios en las personas. (Fibrosis quística1).

Me situaron debajo de un aire acondicionado que me provocó una hemoptisis muy grande, y eso que yo había avisado previamente, pero no me hicieron ni puñetero caso. (Fibrosis quística2).

En una oficina, si la compartes con mucha más gente, cuando hay gripe tienen la mala costumbre, quizás por el sistema en el que vivimos, de que tienen gripe y van a trabajar. Las bajas están mal vistas, entonces ¿qué haces tú? ¿pides tú la baja para que no te contagien? (GD1-Fibrosis quística).

Grado de afectación en la inserción laboral y la vida diaria: en la vida diaria, el 86 \% de la muestra percibe que la fibrosis quística les afecta entre media y muy elevadamente y de manera significativa dependiendo del grado de discapacidad. En el caso de la mujer es mucho más significativa la percepción sobre la afectación (chi-cuadrado es 0,654 ) frente a los hombres, donde no aparece asociarse.

Lo que sucede en la vida cotidiana, como podemos comprobar en la figura 7 , no difiere de lo que acontece en el mundo laboral, donde la mayoría de las respuestas perciben que la fibrosis quística les afecta entre bastante y mucho.

En esta línea, debemos destacar que el hecho de estar trasplantado dificulta aún más la inserción laboral, ya que más del $52 \%$ así lo afirma en el cuestionario.

Además, el $45 \%$ de la muestra sí que percibe que hay discriminación hacia las personas con fibrosis quística en el momento de la contratación. 

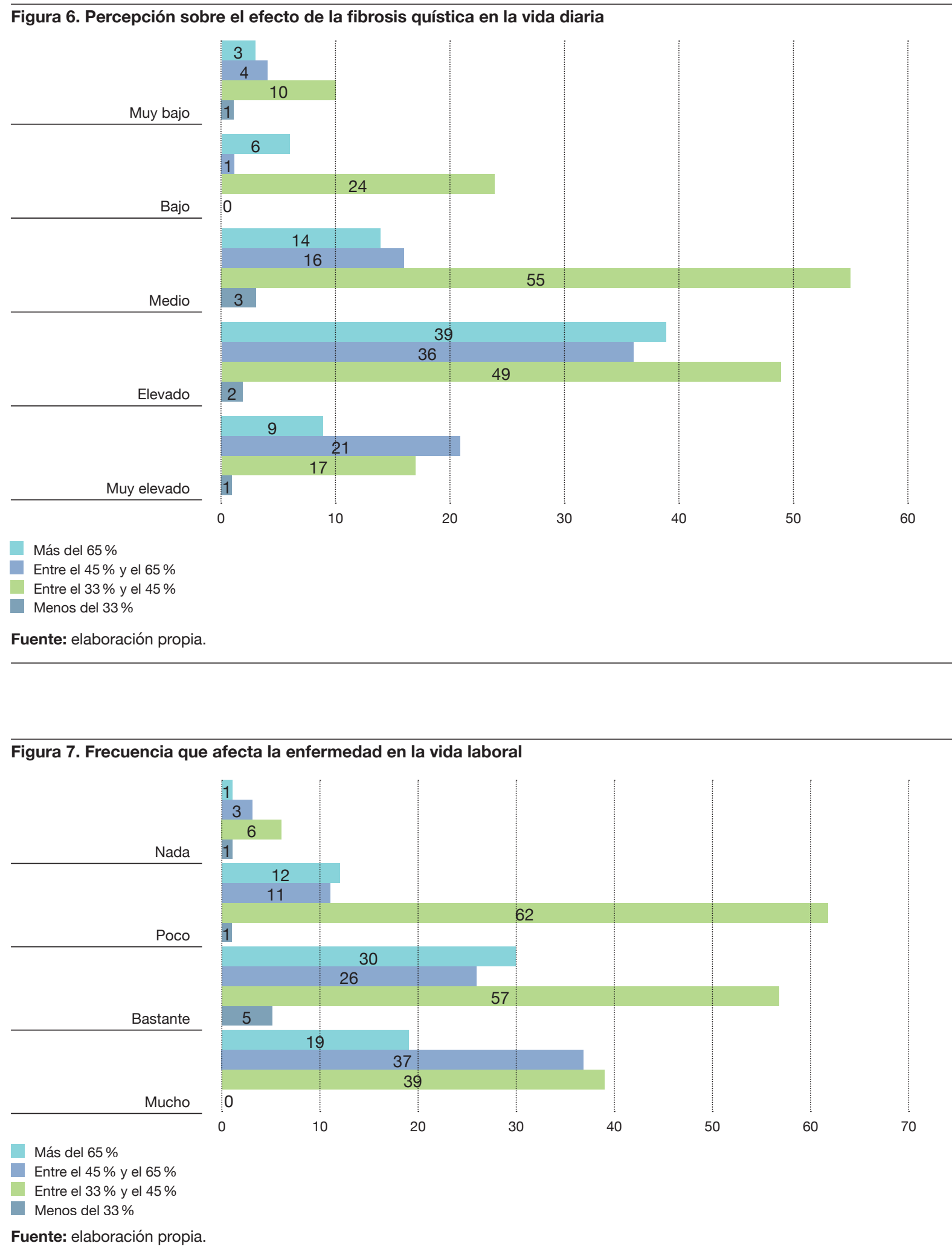
Visibilidad de la fibrosis quística: el factor de que la fibrosis quística no se perciba a simple vista y provoque una discapacidad orgánica era un factor que se quería estudiar para relacionarlo con la discriminación en el entorno laboral o con la desigualdad de oportunidades.

El hecho de que la fibrosis quística no venga acompañada físicamente de elementos visibles supone que la mayoría de los encuestados (81\%) lo identifican como negativo en el ámbito laboral, expresado en que compañeros/as y superiores tengan dificultades para empatizar con el malestar y el cansancio que acompaña la enfermedad.

A ver, te puede ayudar ocultarlo al principio, pero yo creo que luego dificulta. A ti te ven normal y a la hora de pedir un permiso retribuido para ir al médico o tener que coger una baja por un intravenoso, pues no lo entienden. (Fibrosis quística2).

Es un arma de doble filo. Si no se ve, la gente de buenas a primeras no te discrimina. Seguro que a otros con algo más visible como una silla de ruedas o un Down sí les pasa. Pero si te tratan como una persona sana, ponen en ti las expectativas de una persona sana y no las puedes cumplir, entonces cuando pasa algo, no lo entienden y te discriminan porque no has cumplido sus expectativas. (Fibrosis quística3).

El problema que tenemos con la minusvalía es que no se ve, como puede ser el síndrome de Down u otras que a simple vista se ven. La fibrosis quística a simple vista no se ve, y encima si estás trasplantado, te dicen: "huy, si yo te veo muy bien, entonces ¿por qué tienes una minusvalía?... Tienes que explicar por qué vas a faltar al trabajo, por qué tienes que ir a consultas médicas, a pruebas y además el riesgo de coger una gripe o un resfriado que te va a mantener en casa el doble de tiempo de lo normal... y te dicen "vale, ya te llamaré", pero tú sabes que no te van a llamar. (Fibrosis quística4).

Un significativo conjunto de respuestas $(67 \%)$ considera contraproducente en la inserción laboral de las personas con fibrosis quística el hecho de que la comunidad no tenga conocimiento de esta enfermedad.

Creo que el desconocimiento genera prejuicios. Cuando conoces a la persona y conoces su limitación ya no te genera ningún rechazo. Pero plantearlo de buenas a primeras en una entrevista de trabajo genera rechazo, porque $n i$ conoces, ni sabes de qué te habla y puedes pensar "prefiero quitarme el problema de encima porque no se qué me voy a encontrar". (Fibrosis quística1).

Si alguna vez estoy con fiebre y tal me quedo en casa, porque no es lo mismo para mí coger una infección que para otra persona. Pero desde fuera no se ve y, si no se conoce, piensan "¿por qué se tiene que quedar en casa si otros venimos y a cuenta de qué tantos días por una fiebre?”. (Fibrosis quística5).

En el grupo de discusión también se recuperan segmentos narrativos que incardinan en este aspecto.

A los dos meses cogí una baja porque me ingresaron, cuando volví, de repente, de los compañeros no me hablaba nadie. Hacía cosas como "huy, que tos tengo, me voy de baja". Había mucha incomprensión... de los compañeros notaba mucha presión, como es una enfermedad invisible, no lo entendían o no se lo creían... duré 8 meses. (GD4Fibrosis quística).

¿Informar en el centro de trabajo o no informar?: existe una importante prevalencia para informar desde el principio sobre la enfermedad, pero aún así se considera que el tener fibrosis quística puede provocar discriminación. 
En las entrevistas, desde el principio sí puede generar rechazo, pero luego, al saberlo, yo creo que se comprenden más las ausencias... En mi caso, la gente se preocupa más por uno. Por ejemplo, en esta situación actual de COVID, hay compañeros míos que están trabajando y yo estoy teletrabajando, entonces fue al revés, fueron ellos los que fueron a mi despacho y dijeron xxx tienes que irte de aquí ya. (Fibrosis quística1).

Mi oferta fue de empleo protegido, requerían discapacidad, entonces me preguntaron, lo conté y fue genial. (Fibrosis quística2).

El grupo de discusión genera un debate sobre las consecuencias de visibilizar la enfermedad en el centro de trabajo.

Tú tosías y te preguntaban "joder ¿tú estás constipado siempre, ¿eh? Tú el constipado no te lo quitas nunca”, y claro yo explicaba "no, es que tengo fibrosis quística y es esto y tal", y me daba cuenta de que me beneficiaba porque la gente no sabía lo que era, entonces que lo contases les hacía creer lo que creías tú... De 30 personas que éramos no habian oído hablar de fibrosis quística ninguna (GD3-Fibrosis quística).

Sobre todo, si se encadena con bajas y controles muy seguidos, pues la única opción que yo vi posible era contarlo... "pues mira, pasa esto, pero no hay ningún problema, puedo trabajar como nadie y no es contagioso y encima no necesito adaptaciones, sólo ir a revisión de vez en cuando". (GD1-Fibrosis quística).

Oportunidades y adaptaciones: en el grupo de discusión, como tema emergente, aparece la categoría de propuestas, identificadas como oportunidades o adaptaciones que harían que su inserción laboral fuese más fácil, y aparece el teletrabajo como un elemento muy facilitador.

No necesitamos adaptaciones especiales de mobiliario, pero sí esas adaptaciones horarias, que no todas las empresas (por no decir ninguna) están propiciadas a facilitarlas, a no ser que se vean obligadas por ley. (GD4-Fibrosis quística).

Mi función pulmonar cayó en picado, entonces llegó un momento que le pedí a mi jefa teletrabajar, es súper comprensiva. Estuve un par de meses teletrabajando y lo noté mucho, mi trabajo al final es de oficina y en casa el nebulizador te lo puedes poner mientras trabajas y te ahorras una hora de viaje. (GD5-Fibrosis quística).

En mi empresa era inviable teletrabajar hasta que ha venido el COVID-19. Yo lo había solicitado alguna vez, esas veces que entras a las 9.30 pero tienes médico a las 10, pero me dijeron que no... Entonces, cuando empezó lo del COVID-19, dijeron que la gente de riesgo se quedara en casa y le dije a mi jefe "mira, tenemos la opción de que me coja una baja o teletrabaje". Accedió... para mí, genial y él está muy contento, porque saco el mismo trabajo adelante. Espero que toda esta pandemia nos sirva para ver que, desde casa, también la gente rinde... (GD2-Fibrosis quística).

\section{Discusión}

La finalidad de este trabajo es exponer cuál es la situación respecto al ámbito laboral de las personas con fibrosis quística en España y qué elementos pueden favorecerla y obstaculizarla. Desde esta perspectiva, en los resultados se ha podido observar que las personas con fibrosis quística, en la mayoría de los casos, 
pueden acceder al empleo ordinario, aunque tienen dificultades para mantener su continuidad, pero también tienen oportunidades para que la inserción laboral sea una realidad. La elección del tipo de estudios, la invisibilidad de los síntomas, el absentismo laboral, el grado de afectación, la socialización dentro del empleo, serán variables que, según se traten, pueden ser una limitación o no. Además, se han detectado adaptaciones en el entorno laboral que significarían positivamente para el mantenimiento del empleo.

El nivel de estudios de las personas con fibrosis quística es un nivel medio-alto, más del $40 \%$ tiene estudios universitarios. Este dato se valora muy positivamente, ya que el nivel de estudios está asociado a una mayor y más cualificada empleabilidad (Giménez, 2015).

El $75 \%$ de la muestra de este estudio tiene o ha tenido empleo en algún momento de su vida. En un estudio homólogo en Reino Unido de Havermans et al. (2009) aparece un escenario similar, el $70 \%$ de las personas con fibrosis quística estaban empleadas en ese momento y el $94 \%$ lo había estado en algún momento de su vida. Estos porcentajes son superiores al colectivo de personas con el reconocimiento de Certificado de Calificación de Discapacidad en general. En 2019, según el Instituto Nacional de Estadística (2020), la tasa de actividad de las personas con discapacidad se sitúa en el $34 \%$ y la tasa de empleo en el 25,9\%. Si retrocedemos hasta 2016, los porcentajes no suben en ningún momento del 35,2\% y 25,9\%, respectivamente. Donde aparecen las primeras dificultades graves, que rompen con la inserción laboral, son en el mantenimiento de ese puesto de trabajo. El mantener el puesto de trabajo está condicionado por la compatibilidad de las condiciones laborales y la evolución y cuidados de la enfermedad. En uno de los mayores estudios que se ha realizado hasta la fecha sobre empleabilidad y fibrosis quística (Targett et al., 2014), el $47 \%$ de las personas afirmó que la lección de la carrera profesional afectaba a su estado y un $24 \%$ tuvo que cambiar de funciones para poder seguir trabajando.

La elección del tipo de estudios o profesiones no compatibles y el absentismo laboral también se barajan como dificultades de la inserción laboral, según Larborde-Castérot (2012). En concreto, las actividades no recomendadas en los pacientes con fibrosis quística, y por las que las personas de la muestra de este estudio tuvieron que abandonar el empleo fueron, por orden de consenso: trabajo en rama sanitaria, trabajo físico, trabajo con niños, trabajos con exposición al polvo o a humos, trabajos en peluquerías o en contacto con animales. Esta hipótesis se confirma en esta investigación, tal y como se ha comprobado en el verbatio.

No se debería impedir que las personas con fibrosis quística pudieran establecer sus metas profesionales según sus preferencias, pero al mismo tiempo se deben tener en cuenta los propios límites y estar preparados para variar de actividad, si el estado de salud así lo requiere.

La elección del tipo de estudios atendiendo a los condicionantes de la enfermedad no está presente en la mayoría de los casos, a pesar de que ya existen publicaciones e informaciones al colectivo sobre la importancia de elegir profesión teniendo en cuenta las particularidades de la enfermedad (Esteban-Romaní, 2013). Los resultados, tanto cualitativos como cuantitativos, concluyen que mayoritariamente no se han tenido en cuenta las características de la fibrosis quística a la hora de elegir estudios, ni se ha recibido ningún asesoramiento profesional al respecto. Este dato coincide con otros estudios de características similares en otros países (Hogg et al., 2006; Larborde-Castérot, 2012; Zupanic y Skerjanc, 2019).

Se puede concluir que el no tener en cuenta las características derivadas de la fibrosis quística en la elección de estudios produce dificultades añadidas para ejercer la profesión. Un asesoramiento previo a la elección de estudios en adolescentes con fibrosis quística y la motivación hacia estudios superiores sería 
un elemento facilitador para la posterior inserción laboral estable, como se señaló en el grupo de discusión. Y tal y como apuntan Zupanic y Skerjanc (2019), ese asesoramiento debería tener un enfoque multidisciplinario que involucre a progenitores, las y los propios jóvenes con fibrosis quística, personal de psicología y trabajo social y especialistas en el mercado laboral.

Se podía suponer que el grado de afectación percibido y el Certificado de Calificación de Discapacidad afectarían significativamente en la inserción laboral. La estadística sólo correlaciona tímidamente en el caso de las mujeres, en el de los hombres no se correlaciona. Este dato, que a priori puede sorprender, se puede relacionar con otros trabajos: como apunta Hogg et al. (2006), los parámetros clínicos de forma aislada no predicen de manera confiable la discapacidad en el lugar de trabajo. En el estudio de multivariable, Targett et al. (2014) concluye claramente que la afectación general física es menos significativa en la inserción laboral de las personas con fibrosis quística que sus capacidades y motivaciones. La función pulmonar y el nivel educativo son los únicos factores estadísticamente significativos asociados con la situación laboral en fibrosis quística. Este resultado vuelve a demostrar que se debe alentar a los pacientes con fibrosis quística a que alcancen el mayor nivel educativo posible y más adecuado, ya que es probable que este logro determine su empleabilidad tanto o más que su grado de discapacidad, pero ninguno de los trabajos mencionados anteriormente tuvo en consideración la variable sexo.

Otro de los factores que hemos comprobado a lo largo de este trabajo como significativo, en la empleabilidad de las personas con fibrosis quística, es que la discapacidad que produce sea "invisible". El que las limitaciones no se aprecien a simple vista provoca más discriminación y situaciones de incomprensión que si se apreciaran, según el 81 \% de la muestra. El autor Carry Syma (2019) hace un recopilatorio sobre las discapacidades invisibles en el lugar de trabajo: percepciones y barreras razonables. A lo largo de todo el texto constata que hay más reticencia a informar a empleadores y compañeros en las discapacidades invisibles debido a la "ambigüedad de los síntomas" y a que sus adaptaciones pueden ser vistas como "tratos de favor". En la recuperación de segmentos narrativos de esta investigación, se han podido comprobar ejemplos reales al respecto de estos temores. Y en el apartado cuantitativo, los resultados nos muestran que el $45 \%$ considera que la información puede ser un factor discriminador en principio. A ese respecto, en cuanto a si informar o no en el centro de trabajo favorece o dificulta la inserción y estabilidad del empleo, la literatura nos indica en trabajos como los de Modi et al. (2010) que las personas con fibrosis quística son más propensas a informar sobre su enfermedad a familiares y amigos (80\%) que en entornos laborales (39\%). Informan mayormente las personas con enfermedad pulmonar grave, es decir, las que seguramente tengan limitaciones más evidentes. Otro de los factores que intuyen que puede ser determinante para tomar la decisión de informar es la percepción de riesgos y beneficios de la divulgación. Si bien es cierto que, generalmente, las personas que informan manifiestan haber tenido un impacto neutral o positivo. Esta posición se enlaza con el paradigma de falta de sensibilización por desconocimiento. Edwards (2010), en su estudio sobre adultos con fibrosis quística y barreras en el empleo, concluye que la primera barrera es el desconocimiento de los empleadores sobre la enfermedad, que deriva en una posición de perjuicios asociados a la falta de rendimiento y capacidad.

Estos prejuicios y discriminación no suceden en el empleo protegido, que está amparado por la información y la legislación adaptada, por lo que se puede deducir que la información y sensibilización generan entornos "amigables" con la diferencia. $Y$ en el mercado laboral ordinario actual, si no se generan este tipo de entornos será muy difícil que se valoren las capacidades y la calidad, más allá de la cantidad. Las acciones de sensibilización y formación en el entorno laboral son un servicio que se ofrece como proyecto para la inserción laboral de personas con discapacidad en el entorno ordinario (Pallisera et al., 2003). 
Hemos planteado que este trabajo no solo pretendía exponer las limitaciones de inserción, sino también sacar a la luz las adaptaciones que favorecerían la inclusión laboral a largo plazo. En este sentido, flexibilidad horaria y posibilidad de teletrabajo aparecen como las más destacadas.

A lo largo de esta investigación, aparece repetidamente la necesidad de flexibilidad horaria para adaptar los tratamientos de las personas con fibrosis quística al desempeño laboral. En España, el amparo legislativo para realizar ajustes razonables en el mercado laboral ordinario, tal y como reflexiona Gutiérrez (2015), deja fuera a las personas que no tienen la condición de discapacidad reconocida legalmente y "en la práctica" la eficacia de los ajustes o adaptaciones dependen en gran medida del empleador, no así en el empleo protegido para las personas con Certificado de Calificación de Discapacidad. Como apunta Edwards (2010), más allá de nuestras fronteras, para poder mantener su empleo, las personas con fibrosis quística necesitarían flexibilidad en las horas de trabajo, tiempo libre para asistir a citas en el hospital y descansos para llevar a cabo el tratamiento mientras se trabaja. Pero el hecho de si se hacen o no los ajustes parece depender de la voluntad de los empleadores.

Durante la realización de este artículo, en el mundo se declaró la situación de pandemia por COVID-19 y en España se declaró el estado de alarma con confinamiento generalizado. Sólo se podían ejercer presencialmente las profesiones consideradas esenciales. Personas con fibrosis quística de la muestra pasaron a ejercer su profesión en modo teletrabajo, lo que supuso que pudiesen continuar trabajando, lo que de otra manera no hubiese sido posible. El grupo de discusión de esta investigación consensuaba que, para las características de la fibrosis quística, si la profesión lo permite, el teletrabajo resulta una facilidad que hace que se pueda compaginar mucho mejor el mantenimiento de la salud y el ejercicio de la profesión.

Durante este periodo en España no sólo se incorporó el teletrabajo en personas de riesgo por la pandemia, sino también en la mayoría de la población. Desde entonces han corrido ríos de tinta sobre las ventajas y desventajas de esta modalidad. Han aparecido voces que lo tildan de un elemento más de desigualdad de género, ya que provoca sobrecarga en las mujeres que ejercen teletrabajo y además se tienen que ocupar de los cuidados en el hogar a tiempo completo y paralelo. También se le atribuyen desventajas en cuanto a jornadas sin límite y falta desconexión para poder conciliar (De Luis et al., s. f.). A pesar de estas críticas, que si se ponen en contexto se pueden atribuir no sólo al teletrabajo, sino al teletrabajo en situación de confinamiento, hay literatura anterior que lo relaciona directamente como una herramienta de inserción laboral de personas con discapacidad (Salazar, 2007) y le atribuye ventajas como: disminución del absentismo laboral y aumento de la autonomía y flexibilidad.

\section{Conclusiones y propuestas}

A pesar de las limitaciones del estudio, debido a la dificultad de acceso a la muestra (constituida finalmente por personas inscritas en las asociaciones pertenecientes a la Federación Española de Fibrosis Quística), los resultados y posterior discusión llevan a sustentar las siguientes conclusiones con relación a los elementos facilitadores para la inserción laboral de las personas con fibrosis quística:

- Elección de estudios compatibles y motivación a estudios superiores. 
- $\quad$ Flexibilidad en el entorno laboral: horario, teletrabajo, lugar, permisos, etc.

- Información y sensibilización en el mercado laboral ordinario.

- Conocimiento de las características de la enfermedad en la comunidad.

- Legislación de protección a personas con enfermedades crónicas, invisibles y entorno laboral.

En primer lugar, una orientación específica para los adolescentes con fibrosis quística en la elección de estudios será un elemento facilitador para la futura inserción sociolaboral, así como la motivación hacia estudios superiores.

El segundo elemento facilitador sería la flexibilidad horaria y el teletrabajo, como dos adaptaciones que mejorarían la inclusión laboral y el rendimiento de las personas con fibrosis quística.

Un tercer punto precisaría de la sensibilización del entorno laboral y en la comunidad con respecto a las características de la fibrosis quística.

Y finalmente, un elemento fundamental sería la regulación de la legislación, además de la ya existente, para este tema. Es fundamental que los poderes públicos y el desarrollo normativo esté implicado para poder dar cobertura y escenario coherente al entorno que tiene que acoger a las personas con fibrosis quística con y sin Certificado de Calificación de Discapacidad o con otras discapacidades, aunque sean invisibles, y favorecer elementos sociales y económicos que avancen hacia la igualdad de oportunidades.

El grado de afectación o discapacidad que provoque una enfermedad tiene una limitación para desarrollar una profesión, pero también influyen otros factores psicosociales, por lo que las intervenciones, investigaciones y orientaciones en este plano resultarán de gran interés.

Los resultados que se aportan en esta investigación son una contribución interesante a diferentes ámbitos para avanzar en la visibilización de esta enfermedad y sus posibles ajustes favorecedores en la inserción laboral. Es ineludible realizar futuros estudios e intervenciones dirigidas al mercado laboral ordinario para comprobar su punto de vista y vincular ambos con el objetivo de mejorar la empleabilidad de las personas con fibrosis quística. 


\section{Referencias bibliográficas}

Asamblea Médico Mundial (2013). Declaración de Helsinki. Principios éticos para las investigaciones médicas en seres humanos. Asamblea Médico Mundial.

Botija, M. (2016) Trabajo social con personas con diversidad funcional. Tirant lo Blanch.

Callejo, J. (2002). Observación, entrevista y grupo de discusión: el silencio de tres prácticas de investigación. Revista Española de Salud Pública, 76(5), pp. 409-422.

Cortés, F. (2015). Las enfermedades raras. Rev. Med. Clin. Condes, 26(4), pp. 425-431. https://doi.org/10.1016/j. rmclc.2015.06.020.

De Luis, P. et al. (s. f.) El enfoque de género aplicado a la relación teletrabajo medioambiente. http://convega.com/ PortalTele/medioambiente_teletrabajo.pdf.

Demars, N. et al. (2011). Employment experiences among adolescents and young adults with cystic fibrosis. Disabil. Rehabil., 33(11), pp. 922-926. https://doi.org/10.3109/09638288.2010.514644.

Díaz de Rada, V. (2010). Eficacia de las encuestas por internet: un estudio preliminar. Revista Española de Sociología, 13, pp. 49-79.

Edwards, J. y Boxall, K. (2010). Adults with cystic fibrosis and barriers to employment. Disability \& Society, 25(4), pp. 441-453. https://doi.org/10.1080/09687591003755831.

Escobar, J. y Cuervo, A. (2008). Validez de contenido y juicio de expertos: una aproximación a su utilización. Avances en medición, 6(1), pp. 27-36.

España. Real Decreto Legislativo 1/2013, de 29 de noviembre, por el que se aprueba el Texto Refundido de la Ley General de derechos de las personas con discapacidad y de su inclusión social. Boletín Oficial del Estado, 3 de diciembre de 2013, núm. 289, pp. 95635-95673.

Esteban-Legarreta, R. (2021). Discapacidad psíquica "menor", medidas disciplinarias en el marco de una relación de empleo, discriminación y ajustes. Comentario a la sentencia del tribunal constitucional de 15 de marzo de 2021 (STC 51/2021). IUSLabor, 2, pp. 316-330. https://doi.org/10.31009/IUSLabor.2021.i02.11.

Esteban-Romaní, L. (2013). ¿Qué trabajos son perjudiciales para mi enfermedad? En 101 preguntas para fibrosis quística (p. 144). Federación Española de Fibrosis Quística.

Esteban-Romaní, L. (2020). La discapacidad invisible. Relevancia de la figura de la trabajadora social de las asociaciones de pacientes en el reconocimiento de limitaciones y reivindicación de derechos. Caso concreto: fibrosis quística y discapacidad. TS Nova, 16, pp. 75-86.

Federación Española de Fibrosis Quística (2011). Investigación sobre la situación psicosocial de las personas con Fibrosis Quística en España 2011. FEFQ. https://fibrosisquistica.org/wp-content/uploads/2021/03/ investigacio\%CC\%81n-2011_.pdf.

Federación Española de Fibrosis Quística (2021). Tratamiento de la fibrosis quística. Federación Española de Fibrosis Quística. https://fibrosisquistica.org/tratamiento-fibrosis-quistica/.

Giménez, A. R. (2015). Empleabilidad de personas con discapacidad desde el marco rector de SENADIS, Paraguay. Revista Internacional de Investigación en Ciencias Sociales 11(2), pp. 209-222. https://doi.org/10.18004/ riics.2015.diciembre.209-222. 
Gutiérrez, D. (2019). La obligación de realizar ajustes razonables en el puesto de trabajo para personas con discapacidad. Una perspectiva desde el derecho comparado y el derecho español. Bomarzo.

Havermans, T. et al. (2009). Health related quality of life in cystic fibrosis: To work or not to work? Journal of Cystic Fibrosis, 8(3), pp. 218-223. https://doi.org/10.1016/j.jcf.2009.03.002.

Hogg, M. et al. (2006). Work disability in adults with cystic fibrosis and its relationship to quality of life. Journal of Cystic Fibrosis, 6(3), pp. 223-227. https://doi.org/10.1016/j.jcf.2006.10.004.

Instituto Nacional de Estadística (2020) El empleo de las personas con discapacidad. Año 2019. https://www.ine. es/prensa/epd_2019.pdf

Jiménez, A. (2018). Informe Olivenza 2018, sobre la situación general de la discapacidad en España. Observatorio Estatal de la Discapacidad. https://www.cedd.net/es/buscar/Record/541538.

Laborde-Castérot, H. et al. (2011). Employment and work disability in adults with cystic fibrosis. Journal of Cystic Fibrosis, 11(2), pp. 137-143. https://doi.org/10.1016/j.jcf.2011.10.008.

Mercado, E. et al. (2013). Avanzando hacia la igualdad de oportunidades en la inclusión socio-laboral de las personas con discapacidad. Cuadernos de Trabajo Social, 26(1), pp. 95-104. https://doi.org/10.5209/rev_ CUTS.2013.v26.n1.39571.

Modi, A. C. et al. (2010). Assessing disease disclosure in adults with cystic fibrosis: The adult data for understanding lifestyle and transitions (ADULT) survey disclosure of disease in adults with cystic fibrosis. BMC Pulmonary Medicine, 10(46). https://doi.org/10.1186/1471-2466-10-46.

Navarro, J. J. y Pastor, E. (2018). De los riesgos de la socialización. Convergencia Revista de Ciencias Sociales, 76, pp. 119-145.

Pallisera, M. et al. (2003). La integración laboral de las personas con discapacidad en la empresa ordinaria en España. Siglo Cero, 34(4), pp. 5-18.

Salazar, C. (2007). El teletrabajo como aporte a la inserción laboral de personas con discapacidad en Chile: una gran carretera virtual por recorrer. Cienc. Trab., 9(24), pp. 89-98.

Sánchez, A. et al. (2016). Estrategias metodológicas para el estudio de la inserción sociolaboral de las personas con discapacidad. Revista Española de Discapacidad, 4(1), pp. 97-117.

Syma, C. (2019). Invisible disabilities: Perceptions and barriers to reasonable accommodations in the workplace. Library Management, 40(1), pp. 113-120. https://doi.org/10.1108/LM-10-2017-0101.

Targett, K., et al. (2014). Employment in adults with cystic fibrosis. Occupational Medicine, 64(2), pp. 87-94. https:// doi.org/10.1093/occmed/kqt140.

Vázquez, V. (2018). Spanish Cystic Fibrosis Registry: Annual Report 2016. Sociedad Española de Fibrosis Quística. https://fibrosisquistica.org/wp-content/uploads/2018/09/Report2016SpainFinal.pdf.

Zupanic, M. V., y Skerjanc, A. (2019). Cystic fibrosis and carrer counselling. Central European Journal of Public Health, 27(4), pp. 279-284. https://doi.org/10.21101/cejph.a5634. 